\title{
High-Pulsed-Power (49 W) Vertical-Cavity Surface-Emitting Laser with Five Quantum Wells by Uniform Current Injection into Large Emitting Area
}

\author{
Nobuyuki Otake ${ }^{a}$, Eiji Kojima, Hitoshi Yamada, \\ and Yukihiro Takeuchi \\ DENSO Corporation, Research Laboratories \\ 500-1 Minamiyama, Komenoki, Nisshin, Aichi 470-0111, Japan \\ a)nootake@rlab.denso.co.jp
}

\begin{abstract}
We report on the high-power lasing characteristics of a large area bottom-emitting vertical-cavity surface-emitting laser (VCSEL). There have been difficulties in uniform current injection for large area VCSELs, which are caused by the band discontinuity at the interface between AlAs and GaAs of the n-type Distributed Bragg Reflector (DBR). We have reported the n-type DBR using a graded composition interface of $20 \mathrm{~nm}$ thick suppresses the crowding of current to the edge of emitting area and improves external efficiency [1]. The highest peak pulsed power of over $49 \mathrm{~W}$ was achieved by a fivequantum-well VCSEL with a current aperture diameter of $200 \mu \mathrm{m}$.
\end{abstract}

Keywords: VCSEL, DBR, band discontinuity, graded composition Classification: Optoelectronics, Lasers and quantum electronics, Ultrafast optics, Silicon photonics, Planar lightwave circuits

\section{References}

[1] N. Otake, E. Kojima, H. Yamada, and Y. Takeuchi, "High-power large bottom-emitting VCSEL by uniform current injection," to be published in IEICE.

[2] D. Francis, H. L. Chen, W. Yuen, G. Li, and C. Chang-Hansnain, "Monolithic 2D-VCSEL array with $>2 \mathrm{~W}$ CW and $>5 \mathrm{~W}$ pulsed output power," Electron. Lett., vol. 34, no. 22, pp. 2132-2133, Oct. 1988.

[3] M. Miller, M. Grabherr, R. Jager, and K. J. Ebeling, "High-Power VCSEL Arrays for Emission in the Watt Regime at room Temperature," IEEE Photon. Techol. Lett., vol. 3, no. 3, pp. 173-175, March 2001.

[4] T. Li, Y. Ning, Y. Sun, C. Wang, J. Liu, Y. Liu, and L. Wang, "High-power InGaAs VCSEL's single devices and 2-D arrays," J. Lumin., vol. 122, pp. 571-573, Jan.-April 2007.

[5] C. Yan, Y. Ning, L. Qin, S. Zhang, Q. Wang, L. Zhao, Z. Jin, Y. Sun, G. Tao, Y. Liu, G. Chou, L. Wang, and H. Jiang, "A high power InGaAs/GaAsP vertical-cavity surface-emitting laser and its temperature 
characteristics," Semicond. Sci. Technol., vol. 19, pp. 685-689, April 2004.

[6] Y. Zhang, Y. Ning, L. Qin, Y. Wang, J. Cui, G. Liu, X. Zhang, Z. Wang,

Y. Sun, Y. Liu, and L. Wang, "High-power vertical-cavity surface-emitting laser with an optimized p-contact diameter,"

[7] N. Otake, K Abe, H. Yamada, H. Wado, and Y. Takeuchi, "High-Power Vertical-Cavity Surface-Emitting Laser under a Short Pulsed Operation," Appl. Phys. Exp., vol. 2, no. 5, 0521022009.

[8] C. Yan, Y. Ning, L. Qin, D. Cui, Y. Liu, Y. Sun, Z. Jin, H. Li, G. Tao, C. Wang, L. Wang, and H. Jiang, "High-Power Vertical-Cavity SurfaceEmitting Laser With an Extra Au Layer," IEEE Photon. Techol. Lett., vol. 17, no. 8, pp. 1599-1601, Aug. 2005.

\section{Introduction}

Vertical-cavity surface-emitting lasers (VCSELs) are attractive for various applications because of their low cost, ease of designing two-dimensional arrays, and the low divergence angles of the output beam, provided that the issue of their relatively low output power is addressed. The maximum output power of previously reported VCSELs was just over $2 \mathrm{~W}$ for CW operation and just over $20 \mathrm{~W}$ for pulsed operation, both of which were achieved by driving arrayed VCSELs in parallel $[2,3]$. However, a multiple light source array structure is not suitable for applications such as light detection and ranging (LIDAR) as it complicates the optical system for emitting and detecting light in various directions. Therefore a single emitter VCSEL with an output power of over $10 \mathrm{~W}$, which is the typical output power of LIDAR, is needed. The output power of a single emitter VCSEL is limited by thermal rollover caused by Joule heat, so a high power single emitter VCSEL needs to have a low thermal resistance with a large active diameter over $100 \mu \mathrm{m}$ and a bottom-emitting structure $[4,5,6]$. Previously, we reported the maximum output power of $12.5 \mathrm{~W}$ was achieved by a single VCSEL with five quantum well under a pulsed operation with a pulse width of $50 \mathrm{~ns}$ and a repetition rate of $1 \mathrm{kHz}$ [7]. In a large bottom-emitting VCSEL, the active region is close to the p-type electrode and separate from the n-type electrode located on the outside of the emitting area. This causes the non-uniformity of current injection into the active region [8]. Previously we reported that the non-uniformity of current injection was caused by the band discontinuity at the interface between AlAs and GaAs of the n-type Distributed Bragg Reflector (DBR) [1].

The purposes of this study were to achieve high peak pulsed output power from a single VCSEL device under a short-pulsed operation with a pulse width of $10 \mathrm{~ns}$ by suppression of the non-uniformity of current injection and the carrier overflow in active region.

\section{Device structure}

A schematic device structure of our VCSEL, which was produced by metalorganic vapor-phase epitaxy, is shown in Fig. 1. The VCSEL structure 


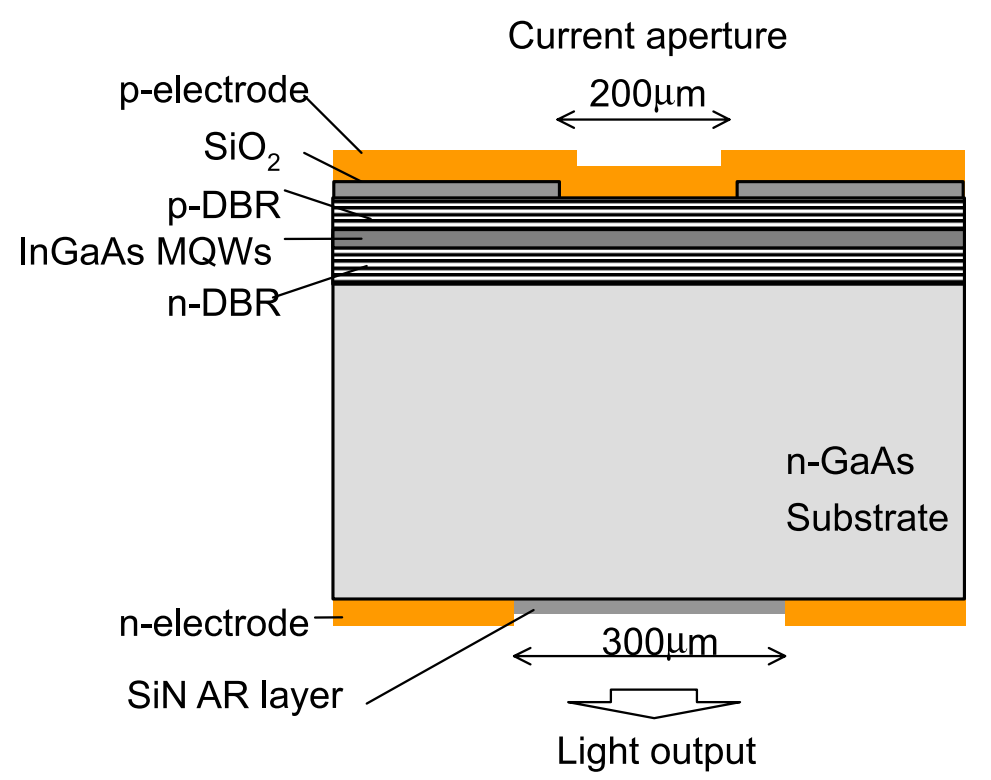

Fig. 1. Schematic device structure of the VCSEL.

consists of the active region sandwiched between 21-period n-type DBR and 25-period p-type DBR. The active region, which contains $6 \mathrm{~nm}$-thick $\mathrm{In}_{0.2} \mathrm{Ga}_{0.8}$ As multiple quantum wells (QWs) separated by $8 \mathrm{~nm}$-thick GaAs barriers, is surrounded by AlGaAs cladding layers to form a one wavelength cavity. Both types of DBR consist of alternating the quarter-wavelength layers of AlAs and GaAs using a linearly graded composition interface which reduces band discontinuity. The p-type electrode $\mathrm{Ti} / \mathrm{Pt} / \mathrm{Au}$ was deposited on the $\mathrm{SiO}_{2}$ passivation layer with a current aperture of $200 \mu \mathrm{m}$. At the bottom of the GaAs substrate, the SiN antireflection layer was deposited with a $300 \mu \mathrm{m}$ diameter. The n-type $\mathrm{AuGe} / \mathrm{Ni} / \mathrm{Au}$ electrode surrounds the $\mathrm{SiN}$ antireflection layer on the bottom of the GaAs substrate.

In this large area bottom-emitting VCSEL, carriers from the p-type electrode, which is located close to the active region, are injected into the active region through the p-type DBR without spreading laterally. On the other hand, carriers from the n-type electrode, which is located away from the active region, spread laterally at the substrate and n-type DBR before reaching the active region. A DBR has a relative large series resistance because of the presence of several tens of hetero-epitaxial layers which act as potential barriers in the hetero interface. Therefore the structure of DBR tends to affect the output power characteristics of the large bottom emitting VCSEL. In order to investigate the dependence of the output power characteristics of VCSELs on the structures of DBRs, VCSELs with the following three types of DBR structures were fabricated; structure (a) contains both types of DBR using $10 \mathrm{~nm}$-thick graded composition interfaces, structure (b) and (d) contains both types of DBR using $20 \mathrm{~nm}$-thick graded composition interfaces, and structure (c) contains p-type DBR using $10 \mathrm{~nm}$-thick graded composition interfaces and n-type DBR using $20 \mathrm{~nm}$-thick graded composition interfaces. The active regions of sturucture (a), (b) and (c) contain InGaAs three QWs, and the active region of structure (d) contains five QWs which supress car- 
reir over flow in the active region,. Specimens of these single VCSELs were operated by a large pulsed current up to $110 \mathrm{~A}$ with a short pulse width of $10 \mathrm{~ns}$ and a repetition rate of $2 \mathrm{kHz}$ at room temperature.

\section{Characteristics for the VCSELs}

Fig. 2 shows the output power characteristics for the VCSELs with various structures. The peak output powers of structure (a), (b), and (c) are $26.1 \mathrm{~W}$, $31.7 \mathrm{~W}$, and $33.8 \mathrm{~W}$, respectively. The difference of these output powers arises from the different DBR structures. The VCSEL containing n-type DBR using the thin graded composition interface tends to lead to output power reduction.

The Near Field Patterns (NFPs) at an injection current of $20 \mathrm{~A}$ are shown in Fig. 3. Structure (a) causes the non-uniformity of the NFP, and structure (b) and (c) suppress the non-uniformity of NFP. The differential resistances of structure (a), (b) and (c) were $0.8 \Omega, 0.6 \Omega$ and $0.6 \Omega$, respectively, The thin

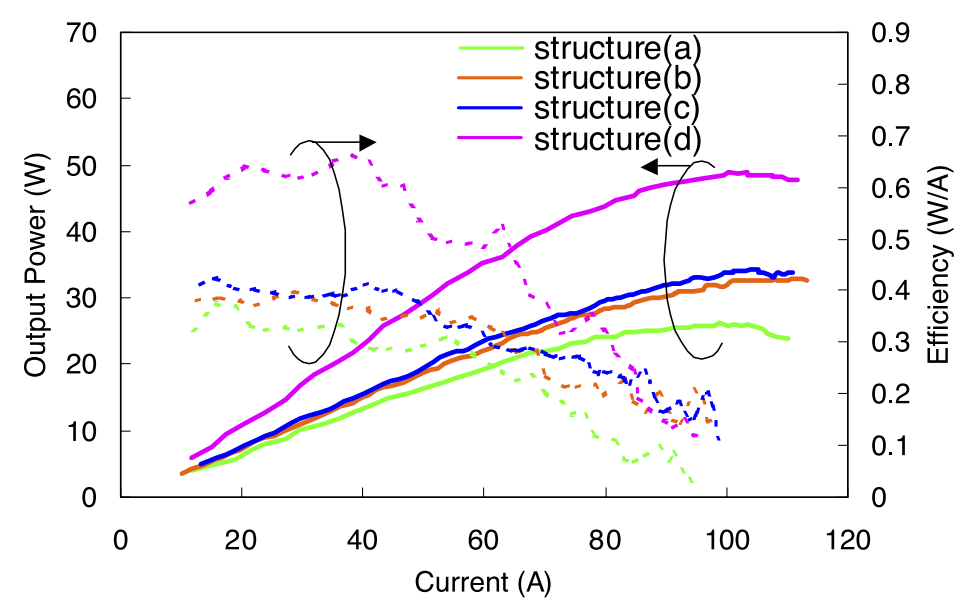

Fig. 2. Output power characteristics of VCSELs with various structures under pulsed operation with a short pulse width of $10 \mathrm{~ns}$ and a repetition rate of $2 \mathrm{kHz}$.

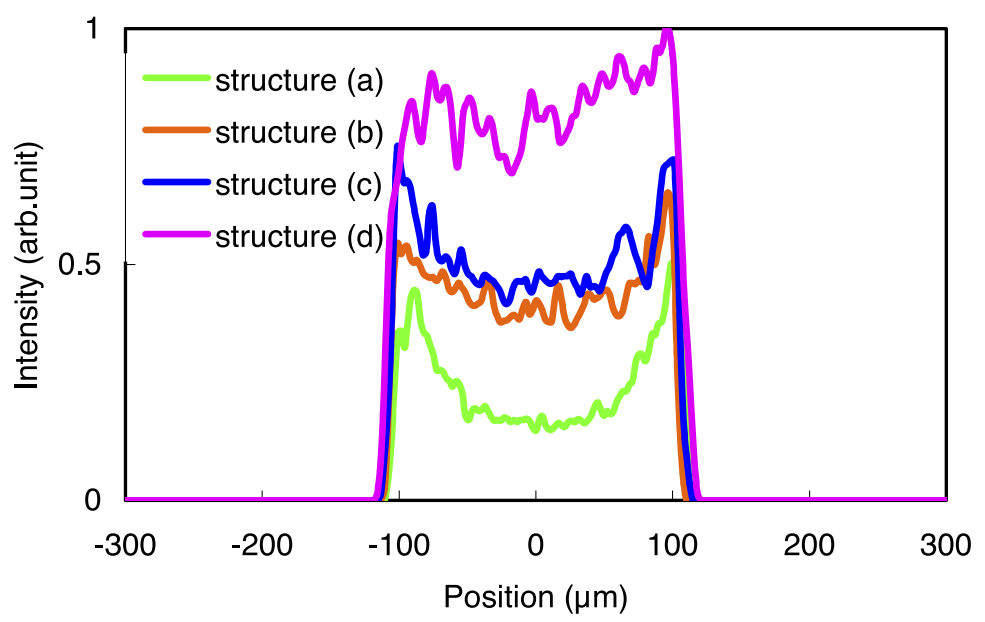

Fig. 3. The Near Field Patterns (NFPs) of VCSELs with various structures at an injection current of $20 \mathrm{~A}$. 
graded composition interface between AlAs and GaAs causes band discontinuity, which increases the resistance of DBRs. For n-type DBR, this band discontinuity inhibits carriers from spreading to the center of the current aperture from the $\mathrm{n}$-type electrode and concentrates carriers in the perimeter of the current aperture. This non-uniformity of the current injection to the active region reduces the output light power. At p-type DBR, the thickness of the graded composition interface between AlAs and GaAs DBR has little effect on the characteristics of the large bottom-emitting VCSEL.

Structure (d) shows higher differential efficiency than the other structures with three QWs. The short-pulsed operation injects numerous carriers into the QWs instantaneously, and causes carrier over flow in the active region. VCSELs with five QWs suppress the carrier overflow. The peak pulsed power of $49 \mathrm{~W}$ at an injection current of $110 \mathrm{~A}$ was achieved by a VCSEL with five QWs due to suppressing the non-uniform carrier injection and the carrier overflow in the active region.

\section{Conclusion}

In summary, we have reported that the large area bottom-emitting VCSEL output power characteristics depend on the thickness of the graded composition interface in the n-type DBR, which causes non-uniformity of current injection into the active region. The VCSEL with the n-type DBR using the $20 \mathrm{~nm}$-thick graded composition interface suppresses the non-uniformity of current injection. A highest peak output power of $49 \mathrm{~W}$ was achieved by a five-quantum-well VCSEL with a $200 \mu \mathrm{m}$ current aperture diameter. This high-peak-power VCSEL is suitable for applications such as LIDAR for long-distance detection.

\section{Acknowledgments}

The authors would like to thank Professor F. Koyama of the Tokyo Institute of Technology for his valuable advice on this research. 